\title{
From Co-algebraic Specifications to Implementation: The Mihda toolkit *
}

\author{
Gianluigi Ferrari, Ugo Montanari, Roberto Raggi, and Emilio Tuosto \\ Dipartimento di Informatica, Universit di Pisa, Italy. \\ \{giangi,ugo, raggi,etuosto\}@di.unipi.it
}

\begin{abstract}
This paper describes the architecture of a toolkit, called Mihda, providing facilities to minimise labelled transition systems for name passing calculi. The structure of the toolkit is derived from the co-algebraic formulation of the partition-refinement minimisation algorithm for HD-automata. HD-automata have been specifically designed to allocate and garbage collect names and they provide faithful finite state representations of the behaviours of $\pi$-calculus processes. The direct correspondence between the coalgebraic specification and the implementation structure facilitates the proof of correctness of the implementation. We evaluate the usefulness of Mihda in practise by performing finite state verification of $\pi$-calculus specifications.
\end{abstract}

\section{Introduction}

Finite state automata (e.g. labelled transition systems) provide a foundational model underlying effective verification techniques of concurrent and distributed systems. From a theoretical point of view, many behavioural properties of concurrent and distributed systems can be naturally defined directly as properties over automata. From a practical point of view, efficient algorithms and verification techniques have been developed and widely applied in practise to case studies of substantial complexity in several areas of computing such as hardware, compilers, and communication protocols. We refer to [2] for a review.

A fundamental property of automata is the possibility, given an automaton, to construct its canonical form: The minimal automaton. The theoretical foundations guarantee that the minimal automaton is indistinguishable from the original one with respect to many behavioural properties (e.g., bisimilarity of automata and behavioural properties expressed in suitable modal or temporal logics). Minimal automata are very important also in practise. For instance, the problem of deciding bisimilarity is reduced to the problem of computing the minimal transition system [8]. Moreover, it is often convenient, from a computational point of view, to verify properties on the minimal automaton rather than on the original one. Indeed, minimisation algorithms can be used to attack

\footnotetext{
* This work has been supported by EU-FET project PROFUNDIS IST-2001-33100 and by MIUR project NAPOLI
} 
state explosion: They yield a small state space, but still retain all the relevant information for the verification.

Global computing systems consists of networks of stationary and mobile components. The primary features of a global computing systems is that components are autonomous, software versioning is highly dynamic, the network coverage is variable and often components reside over the nodes of the network (WEB services), membership is dynamic and often ad hoc without a centralised authority. Global computing systems must be made very robust since they are intended to operate in potentially hostile environments. Moreover, they are hard to construct correctly and very difficult to test in a controlled way: Testers of global computing systems cannot be sure that a system will continue to produce correct results for previously successful test cases. Although significant progresses have been made in providing formal models and effective verification techniques to support verification of global computing systems, current software engineering technologies provide limited solutions to some of the issues discussed above. The problem of formal verification of global computing systems still requires considerable research and dissemination efforts to gain evidence of the viability and weaknesses of the new techniques.

History Dependent automata (HD-automata shortly) have been proposed in $[14,11,4]$ as a new effective model for name passing calculi. Name passing calculi (e.g. the $\pi$-calculus $[10,9,16]$ ) are basically the best known and probably the most acknowledged models of mobility. Moreover, they provide a rich set of techniques for reasoning about mobile systems.

Similarly to ordinary automata, HD-automata are made out of states and labelled transitions; their peculiarity resides in the fact that states and transitions are equipped with names which are no longer dealt with as syntactic components of labels, but become explicit part of the operational model. This allows one to model explicitly name creation/deallocation or name extrusion: These are the distinguished mechanisms of name passing calculi.

HD-automata have been abstractly understood as automata over a permutation model, whose ingredients are a set of names and an action of its group of permutations (renaming substitutions) on an abstract set. This framework is sufficient to describe and reason about formalisms with name-binding operations. It has been incorporated into various kinds of transition systems that aim at providing syntax-free models of name-passing calculi $[5,6,12,15]$

It is important to emphasise that names of states of HD-automata have $l o$ cal meaning. For instance, assume that $A(x, y, z)$ denotes an agent having three (free) names $x, y$ and $z$. Then agent $A(y, x, z)$, obtained through the transformation which swaps names $x$ and $y$, is syntactically different from $A(x, y, z)$. However, these two agents can be semantically represented by means of a single state $q$ of a HD-automaton simply by considering a "swapping" operation on the local names corresponding to names $x$ and $y$. More generally, states that differs only for renaming of their local names are identified in HD-automata. This property allows for a very compact representation of name passing calculi. 
Local meaning of names requires a mechanism for describing how names correspond each other along state transitions. Graphically, we can represent such correspondences using "wires" that connect names of labels, source and target states of transitions. For instance, Figure 1 depicts a transition from source state

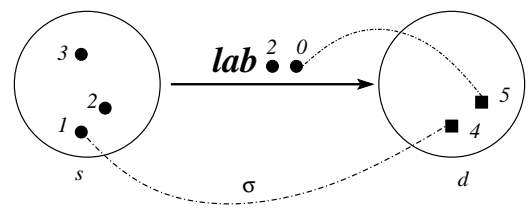

Fig. 1. A HD-automaton transition

$s$ to destination state $d$. The transition exposes two names: Name 2 of $s$ and a fresh name 0 . State $s$ has three names, 1,2 and 3 while state $d$ has two names 4 and 5 which correspond to the old name 1 of $s$ and to the fresh name 0 , respectively. Notice that names 3 is discharged along such state transition.

HD-automata have a natural representation as coalgebras on a category of named sets and named functions. Set elements are equipped with names which are defined up to specific groups of name permutations called symmetries [12]. General results concerning coalgebras guarantees the existence of the minimal HD-automata up to bisimilarity. In [4] two of the authors describe a declarative coalgebraic procedure to perform minimisation of finite state HD-automata.

In this paper, we review the coalgebraic description of the minimisation algorithm for HD-automata, and we illustrate its prototype implementation. This yields a toolkit, called Mihda, providing general facilities to minimise labelled transition systems for name passing calculi. The usefulness of the Mihda toolkit will be shown by performing finite state verification of $\pi$-calculus specifications.

The software architecture of the Mihda toolkit is derived directly from the coalgebraic formulation of the partition-refinement minimisation algorithm. The direct correspondence between the semantical structures and the implementation structures facilitates the design and the implementation of the toolkit. Moreover, it provides the formal machinery to perform the proof of correctness of the implementation.

Recently, several software engineering technologies have been introduced to support a programming paradigm where the web is exploited as a service distributor. By service we do not mean a monolithic web server but rather a component available over the web that others might use to develop other services. Conceptually, web services are stand-alone components that reside over the nodes of the network. Each web service has an interface which is network accessible through standard network protocols and describes the interaction capabilities of the service. The Mihda toolkit have been designed and made available as a WEB service. By a few clicks in a browser at the URL 
http://jordie.di.unipi.it:8080/pweb/ the toolkit can be accessed remotely and its facilities can be evaluated directly over the WEB.

\section{Preliminaries}

This section sketches the main concepts on the coalgebraic representation of automata as a basis for finite state verification by semantic minimisation. We illustrate the approach by providing the coalgebraic specification of the minimisation algorithm for ordinary labelled transition systems. Hereafter, we will use terms 'automaton' and 'labelled transition system' interchangeably.

An automaton $A$ is a triple $(S, L, \rightarrow)$ where $S$ is the set of states, $L$ is the set of actions or labels and $\rightarrow \subseteq S \times L \times S$ is the transition relation. Usually, one writes $s \stackrel{\ell}{\rightarrow} d$ to indicate $(s, \ell, d) \in \longrightarrow ; s$ is the source state and $d$ is the destination or target state.

Let $i d_{A}$ denote the identity function on set $A$ and $f ; g$ be the composition of functions $f$ and $g$ (when it is defined). An endo-functor $\mathcal{F}$ (over category Set) is a mapping from sets to sets and from function to functions that preserves identity functions and function composition. Figure 2 gives a graphical representation of how a functor acts on sets and functions. If $A$ is mapped on $\mathcal{F}(A)$ then $i d_{A}$ is

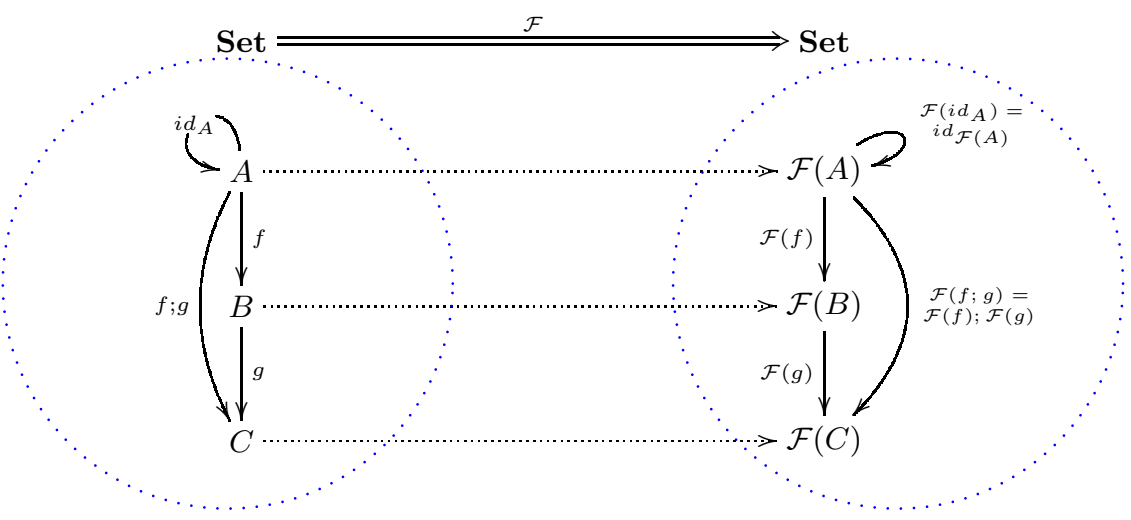

Fig. 2. Functor over Set

associated to $i d_{\mathcal{F}(A)}$ and, if $f$ and $g$ can be composed, then $\mathcal{F}(f)$ and $\mathcal{F}(g)$ can be composed as well. Moreover, the image through $\mathcal{F}$ of the function composition $f ; g$ is obtained by composing the images of functions $f$ and $g$.

Definition 1 ( $\mathcal{F}$-coalgebra). Let $\mathcal{F}$ be an endo-functor on the category Set. $A \mathcal{F}$-coalgebra consists of a pair $(A, \alpha)$ such that $\alpha: A \rightarrow \mathcal{F}(A)$. 
The duality between $\mathcal{F}$-coalgebras and $\mathcal{F}$-algebras (a function $\mathcal{F}(A) \rightarrow A$ ) consists in the fact that domain and codomain are "reversed", namely, are arrows between the same objects but with opposite directions. Different directions can be interpreted as "construction" (induction) and "observation" (coinduction). The interested reader is referred to $[7,1]$.

Before specifying the coalgebraic description of the minimisation algorithm we introduce some notations.

- Expression $Q:$ Set denotes a set and $q: Q$ is synonym of $q \in Q$;

- Fun is the collection of functions among sets (the arrows of category Set).

The function space over sets has the following structure:

$$
\text { Fun }=\{H \mid H=\langle S: \text { Set }, D: \text { Set }, h: S \rightarrow D\rangle\} .
$$

$-h: A \stackrel{b i j}{\longrightarrow} B(h: A \stackrel{i n j}{\longrightarrow} B)$ explicitly states that function $h$ is bijective (injective).

We shall use $S_{H}, D_{H}$ and $h_{H}$ to denote domain, codomain and mapping of an element of Fun,respectively. A similar convention will be used throughout the paper to denote components of tuples.

Let $H, K \in$ Fun be two functions, then the composition of $H$ and $K(H ; K)$ is defined provided that $S_{K}=D_{H}$ and it is the function such that $S_{H ; K}=S_{H}$, $D_{H ; K}=D_{K}$, and $h_{H ; K}=h_{K} \circ h_{H}$. Sometimes, we shall need to work with surjective functions. Hence we let $\widehat{H}$ be the function given by $S_{\widehat{H}}=S_{H}, D_{\widehat{H}}=$ $\left\{q^{\prime}: D_{H} \mid \exists q: S_{H}, h_{H}(q)=q^{\prime}\right\}$ and $h_{\widehat{H}}=h_{H}$.

Finite-state transition systems have been coalgebraically described by employing two ingredients: A set $Q$, that represents the state space, together with a function $K: Q \rightarrow \wp_{\text {fin }}(L \times Q)$ giving the transition relation; $K(q)$ is the set of pairs $\left(\ell, q^{\prime}\right)$ such that $q \stackrel{\ell}{\rightarrow} q^{\prime}$.

In this paper, we shall work on a more concrete representation. In particular, we introduce a mathematical structure, called bundle, whose rôle is to provide a declarative specification of the concrete data structure storing all the transitions out of a given state. Indeed, each bundle details which states are reachable by performing certain actions.

Definition 2 (Bundles). Let $L$ be the set of labels, then a bundle $\beta$ over $L$ is a structure $\left\langle D:\right.$ Set, Step $\left.: \wp_{\text {fin }}(L \times D)\right\rangle$. Set $D$ is the support of $\beta$.

Given a fixed set of labels $L, B^{L}$ denotes the collection of bundles and $\beta: B^{L}$ indicates that $\beta$ is a bundle over $L$.

We now introduce the functor $\mathcal{A}$ over the universe of sets and functions. The following clauses define $\mathcal{A}$ :

- $\mathcal{A}(Q)=\left\{\beta: B^{L} \mid D_{\beta}=Q\right\}$, for each $Q:$ Set;

- For each $H$ : Fun, $\mathcal{A}(H)$ is defined as follows:

- $S_{\mathcal{A}(H)}=\mathcal{A}\left(S_{H}\right)$ and $D_{\mathcal{A}(H)}=\mathcal{A}\left(D_{H}\right)$;

- $h_{\mathcal{A}(H)}: \beta \mapsto\left\langle D_{H},\left\{\left\langle\ell, h_{H}(q)\right\rangle \mid\langle\ell, q\rangle: \operatorname{Step}_{\beta}\right\}\right\rangle$. 
Definition 3 (Transition systems as coalgebras). Let $L$ be a set of labels. Then a labelled transition system over $L$ is a coalgebra for functor $\mathcal{A}$, namely it is a function $K$ such that $D_{K}=\mathcal{A}\left(S_{K}\right)$.

Example 1. A coalgebra $K$ for functor $\mathcal{A}$ represents a transition system where $S_{K}$ is the set of states, and $h_{K}(q)=\beta$, with $D_{\beta}=S_{K}$. Let us consider a finite-state automaton and its coalgebraic formulation via the mapping $h_{K}$.

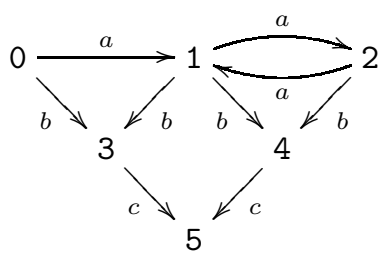

$$
\begin{array}{rlrl}
h_{K}(0) & = & \left\langle S_{k},\right. & \{\langle a, 1\rangle,\langle b, 3\rangle\}\rangle \\
h_{K}(1) & =\left\langle S_{k},\right. & \{\langle a, 2\rangle,\langle b, 3\rangle,\langle b, 4\rangle\}\rangle \\
h_{K}(2) & = & \left\langle S_{k},\{\langle a, 1\rangle,\langle b, 4\rangle\}\right\rangle \\
h_{K}(3) & = & \left\langle S_{k},\{\langle c, 5\rangle\}\right\rangle \\
h_{K}(4) & = & \left\langle S_{k},\{\langle c, 5\rangle\}\right\rangle \\
h_{K}(5) & = & \left\langle S_{k}, \emptyset\right\rangle
\end{array}
$$

Note how, for each state $q \in\{0, \ldots, 5\}, h_{K}(q)$ yields all the immediate successor states of $q$ and the corresponding labels. In other words, $\left(\ell, q^{\prime}\right) \in \operatorname{Step}_{h_{K}(q)}$ if, and only if, $q \stackrel{\ell}{\rightarrow} q^{\prime}$.

General results on coalgebras ensure the existence of the final coalgebra for a large class of functors. These results apply to our formulation of labelled transition systems. In particular, it is interesting to see the result of the iteration along the terminal sequence of functor $\mathcal{A}$.

Let $K$ be a transition system, and let $H_{0}, H_{1}, \ldots, H_{i+1}, \ldots$ be the sequence of functions computed by

$$
H_{i+1}=K ; \widehat{\mathcal{A}\left(H_{i}\right)},
$$

where $H_{0}$ is the unique function from $S_{K}$ to the one-element set $\{*\}$ given by $S_{H_{0}}=S_{K} ; D_{H_{0}}=\{*\} ;$ and $h_{H_{0}}\left(q: S_{H_{0}}\right)=*$.

Finiteness of $\wp_{\text {fin }}$ ensures convergence of the iteration along the terminal sequence. We can say much more if the transition system is finite state. Indeed, if $K$ is a finite-state transition system, then

- The iteration along the terminal sequence converges in a finite number of steps, i.e. $D_{H_{n+1}} \equiv D_{H_{n}}$ (for some natural number $n$ ),

- The isomorphism mapping $F: D_{H_{n}} \rightarrow D_{H_{n+1}}$ yields the minimal realisation of transition system $K$.

Comparing the co-algebraic construction with the standard algorithm $[8,3]$ which constructs the minimal labelled transition system we can observe:

- at each iteration $i$ the elements of $D_{H_{i}}$ are the blocks of the minimisation algorithm (i.e. the $i$-th partition). Notice that the initial approximation $D_{H_{0}}$ contains a single block: in fact $H_{0}$ maps all the states of the transition system into $\{*\}$.

- at each step the algorithm creates a new partition by identifying the splitters for states $q$ and $q^{\prime}$. This corresponds in our co-algebraic setting to the fact that $H_{i}(q)=H_{i}\left(q^{\prime}\right)$ but $H_{i+1}(q) \neq H_{i+1}\left(q^{\prime}\right)$. 
- the iteration proceeds until a stable partition of blocks is reached: then the iteration along the terminal sequence converges.

We now apply the iteration along the terminal sequence to the coalgebraic formulation of the transition system of Example 1. The initial approximation is the function $H_{0}$ defined as follows $H_{0}=\left\langle S_{H_{0}}=S_{K}, D_{H_{0}}=\{*\}, h_{H_{0}}(q)=*\right\rangle$ and the first approximation $H_{1}$ is the map $h_{H_{1}}: q \mapsto\left\langle D_{H_{0}},\left\{\left\langle\ell, h_{H_{0}}\left(q^{\prime}\right)\right\rangle: q \stackrel{\ell}{\rightarrow} q^{\prime}\right\}\right\rangle$ obtained by

$$
T\left(H_{0}\right)\langle\{1,2,3,4,5\},\{\langle a, 2\rangle,\langle b, 3\rangle,\langle b, 4\rangle\}\rangle=\langle\{*\},\{\langle a, *\rangle,\langle b, *\rangle\}\rangle
$$

We obtain the function $h_{H_{1}}$ and the destination state $D_{H_{1}}=\left\{\beta_{1}, \beta_{2}, \beta_{3}\right\}$ as detailed below.

$$
\begin{aligned}
& h_{H_{1}}(0)=\langle\{*\},\{\langle a, *\rangle,\langle b, *\rangle\}\rangle \\
& h_{H_{1}}(1)=\langle\{*\},\{\langle a, *\rangle,\langle b, *\rangle\}\rangle \\
& h_{H_{2}}(2)=\langle\{*\},\{\langle a, *\rangle,\langle b, *\rangle\}\rangle \quad \beta_{1}=\langle\{*\},\{\langle a, *\rangle,\langle b, *\rangle\}\rangle \\
& h_{H_{1}}(3)=\quad\langle\{*\},\{\langle c, *\rangle\}\rangle \quad \beta_{2}=\quad\langle\{*\},\{\langle c, *\rangle\}\rangle \\
& h_{H_{1}}(4)=\langle\{*\},\{\langle c, *\rangle\}\rangle \\
& h_{H_{1}}(5)=\quad\langle\{*\}, \emptyset\rangle
\end{aligned}
$$

A further iteration yields:

$$
\begin{array}{rrr}
h_{H_{2}}(0) & =\left\langle D_{H_{1}},\left\{\left\langle a, \beta_{1}\right\rangle,\left\langle b, \beta_{2}\right\rangle\right\}\right\rangle \\
h_{H_{2}}(1) & =\left\langle D_{H_{1}},\left\{\left\langle a, \beta_{1}\right\rangle,\left\langle b, \beta_{2}\right\rangle\right\}\right\rangle \\
h_{H_{2}}(2) & =\left\langle D_{H_{1}},\right. & \left.\left\{\left\langle a, \beta_{1}\right\rangle,\left\langle b, \beta_{2}\right\rangle\right\}\right\rangle \\
h_{H_{2}}(3)= & \left\langle D_{H_{1}},\{\langle c, \emptyset\rangle\}\right\rangle \\
h_{H_{2}}(4)= & \left\langle D_{H_{1}},\{\langle c, \emptyset\rangle\}\right\rangle \\
h_{H_{2}}(5)= & \left\langle D_{H_{1}}, \emptyset\right\rangle
\end{array}
$$

Since $D_{H_{2}} \equiv D_{H_{1}}$ the iterative construction converges, thus providing the minimal labelled transition system depicted as

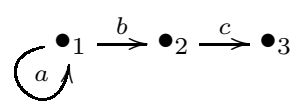

where $\bullet_{1}=\{0,1,2\}, \bullet_{2}=\{3,4\}$ and $\bullet_{3}=\{5\}$.

\section{HD-automata for $\pi$-agents}

This section outlines the representation of HD-automata as coalgebras over the concrete permutation algebra of named sets.

Let $\mathcal{N}$ be an infinite countable set of names ranged over by $v$ and let $\mathcal{N}^{*}$ be the set $\mathcal{N} \cup *$, where $* \notin \mathcal{N}$ is a distinguished name and will be used for modelling name creation. We also assume that $<$ is a total order on $\mathcal{N}^{\star}$ (for instance, it can be the lexicographic order on $\mathcal{N}$ and $\forall v \in \mathcal{N}: *<v$ ).

Table 1 displays the definitions of named sets, named functions, and composition of named functions. In Table 1, the general product $\prod$ is employed (as usual in type theory) to type functions $f$ such that the type of $f(q)$ is dependent on 
Named set A named set $A$ is a structure

$$
A=\left\langle Q: \operatorname{Set},\left|{ }_{-}\right|: Q \longrightarrow \omega, \leq: \wp(Q \times Q), G: \prod_{q \in Q} \wp\left(\left\{v_{1} . . v_{|q|}\right\} \stackrel{b i j}{\longrightarrow}\left\{v_{1} . . v_{|q|}\right\}\right)\right\rangle
$$

where $\forall q: Q_{A}, G_{A}(q)$ is a permutation group and $\leq_{A}$ is a total ordering.

Named function A named function $H$ is a structure

$$
H=\left\langle S: \text { NSet }, D: \text { NSet }, h: Q_{S} \longrightarrow Q_{D}, \Sigma: Q_{S} \longrightarrow \wp\left(\{h(q)\}_{D} \stackrel{i n j}{\longrightarrow}\{q\}_{S}\right)\right\rangle
$$

where $\forall q: Q_{S_{H}}, \forall \sigma: \Sigma_{H}(q)$,

1. $G_{D_{H}}\left(h_{H}(q)\right) ; \sigma=\Sigma_{H}(q)$ and

2. $\sigma ; G_{S_{H}}(q) \subseteq \Sigma_{H}(q)$.

Composition of named functions Named functions can be composed in the obvious way. Let $H$ and $K$ be named functions. Then $H ; K$ is defined only if $D_{H}=S_{K}$, and

$$
\begin{gathered}
S_{H ; K}=S_{H}, \quad D_{H ; K}=D_{K}, \quad h_{H ; K}: Q_{S_{H}} \longrightarrow Q_{D_{K}}=h_{H} ; h_{K}, \\
\Sigma_{H ; K}\left(q: Q_{S_{H}}\right)=\Sigma_{K}\left(h_{H}(q)\right) ; \Sigma_{H}(q)
\end{gathered}
$$

Let $H$ be a named function, $\widehat{H}$ denotes the surjective component of $H$ :

$-S_{\widehat{H}}=S_{H}$ and $Q_{D_{\widehat{H}}}=\left\{q^{\prime}: Q_{D_{H}} \mid \exists q: Q_{S_{H}} \cdot h_{H}(q)=q^{\prime}\right\}$,

$-|q|_{D_{\widehat{H}}}=|q|_{D_{H}}, G_{D_{\widehat{H}}}^{H_{\widehat{H}}}(q)=G_{D_{H}}(q), h_{\widehat{H}}(q)=h_{H}(q)$ and $\Sigma_{\widehat{H}}(q)=\Sigma_{H}(q)$

Table 1. Named sets, Named Functions and Composition of Named Functions

$q$. Intuitively, a named set represents a set of states equipped with a mechanism to give local meaning to names occurring in each state. In particular, function | - | yields the number of local names of states. Moreover, the permutation group $G_{A}(q)$ allows one to describe directly the renamings that do not affect the behaviour of $q$, i.e., symmetries on the local names of $q$. For technical reasons, we assume that states are totally ordered.

By convention we write $\left\{q: Q_{A}\right\}_{A}$ to indicate the set $\left\{v_{1}, \ldots, v_{|q|_{A}}\right\}$ and we use NSet to denote the universe of named sets.

As in the case of standard transition systems, named functions are used to determine the possible transitions of a given state. Intuitively, $h_{H}(q)$ yields the behaviour of state $q: S_{H}$, i.e. the transitions departing from $q$. Since states are equipped with local names, a name correspondence is needed to describe how names in the destination state are mapped into names of the source state, therefore we must equip $H$ with a set $\Sigma_{H}(q)$ of injective functions. However, names of corresponding states $\left(q, h_{H}(q)\right)$ in $h_{H}$ are defined up to permutation 
groups and name correspondence must not be "sensible" to the local meaning of names. Therefore, the whole set $\Sigma_{H}(q)$ must be generated by saturating any of its elements by the permutation group of $h_{H}(q)$, and the result must be invariant with respect to the permutation group of $q$. Condition (1) in Table 1 states that the group of $h_{H}(q)$ does not change the meaning of names in $h_{H}(q)$, while Condition (2) states that the group of $q$ does not "generate meanings" for local names of $q$ that are outside $h_{H}(q)$.

\subsection{Bundles over $\pi$-calculus actions}

To represent the minimisation algorithm for the early semantics of $\pi$-calculus [10], the notion of bundle must be enriched. Labels of transitions must distinguish among the different meanings of names occurring in $\pi$-calculus actions, namely synchronisation, bound/free output and bound/free input. The set of $\pi$-calculus labels $L_{\pi}$ is $\{T A U, B O U T, O U T, B I N, I N\}$. We specify two different labels for input actions: Label $B I N$ is used when the input transition exposes a fresh name, while label $I N$ handles the case of an input transition that exposes a name of the source state of the transition. Labels in $L_{\pi}$ have weights. The weight map |-| $: L_{\pi} \rightarrow\{\emptyset,\{1\},\{1,2\}\}$ is defined as:

$$
|T A U|=\emptyset \quad|B O U T|=|B I N|=\{1\} \quad|O U T|=|I N|=\{1,2\}
$$

and associates the set of indexes of distinct names the label refers to.

A bundle on $\pi$-labels is defined as in Table 2 . Table 2 illustrates definitions of bundles and names of bundles. As it is the case for ordinary automata, the Step component of a bundle declares the set of successor states for a given source state. More precisely, if $\langle\ell, \pi, \sigma, q\rangle \in q d \mathcal{D}$, then $q$ is the destination state; $\ell$ is the label of the transition; $\pi$ associates to the label the names observed in the transition; and $\sigma$ states how names in the destination state are related to the names in the source state. According to the definition of $\sigma$ in Table 2, a name in a destination state of a quadruple is mapped on the distinguished name $*$ only on transitions where a new name is created (i.e. transitions labelled by BOUT or $B I N)$.

In order to exploit named functions for representing HD-automata it is necessary to equip the set of bundles $B$ with a named set structure. In other words we must define

- a total order on bundles,

- a function that maps a bundle to its number of names,

- a group of permutations over those names.

The names of a bundle are the names (different from $*$ ) that appear either in the labels or in the range of $\sigma$ 's of the quadruples of the bundle. Without loss a generality, we can assume that a total order on states and labels exist. Hence, quadruples are totally ordered ${ }^{1}$. The order over quadruples yields an ordering $\sqsubseteq$ over bundles.

\footnotetext{
${ }^{1}$ For instance, we can assume the lexicographic order of labels, states and names.
} 
Bundles A bundle $\beta$ consists of the structure

$$
\beta=\langle\mathcal{D}: \text { NSet, Step }: \wp(q d \mathcal{D})\rangle
$$

where $q d \mathcal{D}$ is the set of quadruples of the form $\langle\ell, \pi, \sigma, q\rangle$ given by

$$
q d \mathcal{D}=\left\{\left\langle\ell: L_{\pi}, \pi: \mathcal{N} \stackrel{i n j}{\longrightarrow}\left\{v_{1} . .\right\}, \sigma: \prod_{\ell \in L_{\pi}}\{q\}_{\mathcal{D}} \stackrel{i n j}{\rightarrow} Q \ell, q: Q_{\mathcal{D}}\right\rangle\right\}
$$

and

$$
Q \ell= \begin{cases}\mathcal{N}^{\star} & \text { if } \ell \in\{B O U T, B I N\} \\ \mathcal{N} & \text { if } \ell \notin\{B O U T, B I N\}\end{cases}
$$

under the constraint that $G_{\mathcal{D}_{\beta}}(q) ; S_{q}=S_{q}$, where $S_{q}=\left\{\langle\ell, \pi, \sigma, q\rangle \in\right.$ Step $\left._{\beta}\right\}$ and $\rho ;\langle\ell, \pi, \sigma, q\rangle=\langle\ell, \pi, \rho ; \sigma, q\rangle$.

Bundle names Let $\beta$ be a bundle. Function $\{|-|\}: B \rightarrow \mathcal{N}$, mapping each bundle to the set of its names, is defined by

$$
\{\beta \mid\}=\bigcup_{\langle\ell, \pi, \sigma, q\rangle \in \text { Step }_{\beta}} \operatorname{rng}(\pi) \cup r n g(\sigma) \backslash\{*\}
$$

where $r n g$ yields the range of functions. We only consider bundles $\beta$ such that $\{|\beta|\}$ is finite and we let $\lfloor\beta\rfloor$ to indicate the number of names which occur in the bundle $\beta$ (i.e. $\lfloor\beta\rfloor=|\{\beta \mid\}|)$.

Table 2. Bundles: the $\pi$-calculus case

The group of $\beta: B^{L_{\pi}}$ is the set of permutations $\theta:\{|\beta|\} \stackrel{b i j}{\longrightarrow}\{|\beta|\}$ such that $\beta ; \theta=\beta$ where $\beta ; \theta$ is defined as $\left\langle\mathcal{D}_{\beta},\{\langle\ell, \pi ; \theta, \sigma ; \theta, q\rangle \mid\langle\ell, \pi, \sigma, q\rangle: \beta\}\right\rangle$.

\subsection{Normalising bundles}

In the minimisation algorithm two states belong to the same block (partition) whenever they have the "same" bundles. Hence, the most crucial construction on bundles is the normalisation operation. This operation is necessary for two different reasons. The first reason is that there are different equivalent ways for picking up the step components (i.e. quadruples $\langle\ell, \pi, \sigma, q\rangle$ ) of a bundle. The second (more important) reason is for removing from the step component of a bundle all the redundant input transitions. Indeed, redundant transitions occur when a HD-automaton is built from a $\pi$-calculus agent. During this phase, it is not possible to decide which free input transitions are required, and which transitions are covered by the bound input transition ${ }^{2}$. The solution to this problem

${ }^{2}$ In the general case, to decide whether a free input transition is required it is as difficult as to decide the bisimilarity of two $\pi$-calculus agents. 
consists of adding a superset of the required free input transitions and to exploit a reduction function to remove the unnecessary ones during the minimisation phase. Consider for instance the case of a state $q$ having only one name $v_{1}$ and assume that the following two tuples appear in a bundle:

$$
\left\langle I N, x y,\left\{v_{1} \rightarrow y\right\}, q\right\rangle \quad \text { and } \quad\left\langle B I N, x,\left\{v_{1} \rightarrow *\right\}, q\right\rangle .
$$

Then, the $I N$ transition is redundant if $y$ is not active in $q$ as it expresses exactly the same behaviour of the second tuple, except that a "free" input transition is used rather than a "bound" one. Hence, the transformation removes the first tuple from the bundle. During the iterative execution of the minimisation algorithm, bundles are split; hence the set of redundant transitions of bundles decreases. Hence, when the iterative construction terminates, only those free inputs that are really redundant have been removed from the bundles.

The normalisation of a bundle $\beta$ is done in different steps. First, the bundle is reduced by removing all the possibly redundant input transitions. Reduction function $\operatorname{red}(\beta)$ on bundles is defined as follows:

$-\mathcal{D}_{\text {red }(\beta)}=\mathcal{D}_{\beta}$

- Step $\operatorname{red}(\beta)=\operatorname{Step}_{\beta} \backslash\left\{\langle I N, x y, \sigma, q\rangle \mid\left\langle B I N, x, \sigma^{\prime}, q\right\rangle: \operatorname{Step}_{\beta} \wedge \sigma^{\prime}=\sigma ;\{y \rightarrow *\}\right\}$.

where $\sigma ;\{y \rightarrow *\}$ is the function equal to $\sigma$ on any name different from $y$ and that assigns $*$ to $y$. Second, the normalisation function $n o r m(\beta)$ is defined as follows:

$-\mathcal{D}_{\text {norm }(\beta)}=\mathcal{D}_{\beta}$

$-\operatorname{Step}_{n o r m(\beta)}=\min _{\sqsubseteq}\left(\operatorname{Step}_{\beta} \backslash\left\{\langle I N, x y, \sigma, q\rangle \mid y \notin a n_{\beta}\right\}\right)$,

where $a n_{\beta}=\{|\operatorname{red}(\beta)|\}$ is the active names of $\beta$ and $m i n_{\sqsubseteq}$ is the function that, when applied to $\mathrm{Step}_{\beta}$, returns the step of the minimal bundle (with respect to order $\sqsubseteq$ ) among those obtained by permuting names of $\beta$ in all possible ways $^{3}$. The order relation $\sqsubseteq$ is used to define the canonical representatives of bundles and relies on the order of quadruples. For this reason we introduced an ordering relation on named sets in the first place. In the following, we use $\operatorname{perm}(\beta)$ to denote the canonical permutation that associates $\operatorname{Step}_{\text {norm }(\beta)}$ and Step $_{\beta} \backslash\left\{\langle I N, x y, \sigma, q\rangle \mid y \notin a n_{\beta}\right\}$.

We remark that, while all $I N$ transitions covered by $B I N$ transitions are removed in the definition of $\operatorname{red}(\beta)$, only those corresponding to the reception of non-active names are removed in the definition of $\operatorname{norm}(\beta)$. In fact, even if an input transition is redundant, it might be the case that it corresponds to the reception of a name that is active due to some other transitions.

Finally, we need a construction which extracts in a canonical way a group of permutations out of a bundle. Let $\beta$ be a bundle, define $G r \beta$ to be the set $\left.\left\{\rho \mid \operatorname{Step}_{\beta} ;\left(\rho^{*} / *\right]\right)=\operatorname{Step}_{\beta}\right\}$. It can be proved that $\operatorname{Gr} \beta$ is a group of permutations.

\footnotetext{
$\overline{{ }^{3} \text { More precisely, given a bundle } \bar{\beta}, \min } \bar{\sqsubseteq}$ is the minimal bundle in the set $\{\bar{\beta} ; \theta \mid \theta$ : $\{\bar{\beta} \mid\} \stackrel{b i j}{\longrightarrow}\{|\bar{\beta}|\}\}$, with respect to the total ordering $\sqsubseteq$ of bundles over $\mathcal{D}$.
} 


\subsection{The minimisation algorithm}

We are now ready to give the definition of the functor $\mathcal{T}$ that states the coalgebras for HD-automata. The action of functor $\mathcal{T}$ over named sets is given by:

- $Q_{\mathcal{T}(A)}=\left\{\beta:\right.$ Bundle $\mid \mathcal{D}_{\beta}=A, \beta$ normalised $\}$,

$-|\beta|_{\mathcal{T}(A)}=\lfloor\beta\rfloor$,

$-G_{\mathcal{T}(A)}(\beta)=G r \beta$

$-\beta_{1} \leq_{\mathcal{T}(A)} \beta_{2}$ iff Step $_{\beta_{1}} \sqsubseteq$ Step $_{\beta_{2}}$,

while the action of functor $\mathcal{T}$ over named functions is given by:

$-S_{\mathcal{T}(H)}=\mathcal{T}\left(S_{H}\right), D_{\mathcal{T}(H)}=\mathcal{T}\left(D_{H}\right)$,

$-h_{\mathcal{T}(H)}\left(\beta: Q_{\mathcal{T}\left(S_{H}\right)}\right): Q_{\mathcal{T}\left(D_{H}\right)}=\operatorname{norm}\left(\beta^{\prime}\right)$,

- $\Sigma_{\mathcal{T}(H)}\left(\beta: Q_{\mathcal{T}\left(S_{H}\right)}\right)=\operatorname{Gr}\left(\right.$ norm $\left.\left(\beta^{\prime}\right)\right) ;\left(\operatorname{perm}\left(\beta^{\prime}\right)\right)^{-1} ; \operatorname{inj}:\left\{n \operatorname{norm}\left(\beta^{\prime}\right)\right\} \longrightarrow\{\beta\}_{\mathcal{T}\left(S_{H}\right)}$ where $\beta^{\prime}=\left\langle D_{H},\left\{\left\langle\ell, \pi, \sigma^{\prime} ; \sigma, h_{H}(q)\right\rangle \mid\langle\ell, \pi, \sigma, q\rangle: \operatorname{Step}_{\beta}, \sigma^{\prime}: \Sigma_{H}(q)\right\}\right\rangle$.

Notice that functor $\mathcal{T}$ maps every named set $A$ into the named set $\mathcal{T}(A)$ of its normalised bundles. A named function $H$ is mapped into a named function $\mathcal{T}(H)$ in such a way that every corresponding pair $\left(q, h_{H}(q)\right)$ in $h_{H}$ is mapped into a set of corresponding pairs $\left(\beta, \operatorname{norm}\left(\beta^{\prime}\right)\right)$ of bundles in $h_{\mathcal{T}(H)}$. The quadruples of bundle $\beta^{\prime}$ are obtained from those of $\beta$ by replacing $q$ with $h_{H}(q)$ and by saturating with respect to the set of name mappings in $\Sigma_{H}(q)$. The name mappings in $\Sigma_{\mathcal{T}(H)}(\beta)$ are obtained by transforming the permutation group of bundle $\operatorname{norm}\left(\beta^{\prime}\right)$ with the inverse of the canonical permutation of $\beta^{\prime}$ and with a fixed injective function inj mapping the set of names of norm $\left(\beta^{\prime}\right)$ into the set of names of $\beta$, defined as $i<j, \operatorname{inj}\left(v_{i}\right)=v_{i^{\prime}}$ and $i n j\left(v_{j}\right)=v_{j^{\prime}}$ implies $i^{\prime}<j^{\prime}$. Without bundle normalisation, the choice of $\beta^{\prime}$ among those in $\beta^{\prime} ; \theta$ would have been arbitrary and not canonical with the consequence of mapping together fewer bundles than needed.

Definition 4 (Transition systems for $\pi$-agents). A transition system over named sets and $\pi$-actions is a named function $K$ such that $D_{K}=\mathcal{T}\left(S_{K}\right)$.

HD-automata are particular transition systems over named sets. An HD-automaton $A$ is given by:

- the elements of $Q_{A}$ are $\pi$-agents and $\leq_{A}$ is the lexicographic order on $Q_{A}$;

$-\left|p\left(v_{1}, \ldots, v_{n}\right)\right|_{A}=n$;

- $G_{A}(q)=\left\{i d:\{q\}_{A} \longrightarrow\{q\}_{A}\right\}$, where $i d$ denotes the identity function,

$-h: Q_{A} \longrightarrow\left\{\beta \mid \mathcal{D}_{\beta}=A\right\}$ is such that $\left\langle\ell, \pi, \sigma, q^{\prime}\right\rangle \in \operatorname{Step}_{h(q)}$ represent the $\pi$-calculus transitions from agent $q$.

We will often use the notation $q \stackrel{\ell, \pi, \sigma}{\longrightarrow} q^{\prime}$ to denote the "representative" transitions from agent $q$ that are used in the construction of the HD-automaton.

We can now define the function $K$.

$$
\begin{aligned}
& -S_{K}=A, \\
& -h_{K}(q)=\operatorname{norm}(h(q)), \\
& -\Sigma_{K}(q)=\operatorname{Gr}\left(h_{K}(q)\right) ;(\operatorname{perm}(h(q)))^{-1} ; i n j:\{\mid h(q)\} \longrightarrow\{q\}_{A}
\end{aligned}
$$


The minimal HD-automata is built by an iterative procedure on $K$ : the iteration along the terminal sequence. The formula which details the iterative construction is given by

$$
H_{i+1}=K ; \widehat{\mathcal{T}\left(H_{i}\right)} .
$$

If $K$ is a finite state HD-automaton. Then

The initial approximation, $H_{0}$, is defined as follows:

$-S_{H_{0}}=S_{K}, D_{H_{0}}=$ unit where $Q_{\text {unit }}=\{*\},|*|_{\text {unit }}=0$ (and hence $\left.\{*\}=\phi\right)$, $G_{\text {unit }} *=\phi$, and $* \leq_{\text {unit }} *$,

$-h_{H_{0}}\left(q: Q_{s_{H_{0}}}\right)=*$,

$-\Sigma_{H_{0}}(q)=\{\phi\}$

We recall that the iteration along the terminal sequence converges in a finite number of steps: $i$ exists such that $D_{H_{i+1}} \equiv D_{H_{i}}$, and the isomorphism mapping $F: D_{H_{i}} \rightarrow D_{H_{i+1}}$ yields the minimal realisation of the transition system $K$ up to strong early bisimilarity.

\section{The Mihda toolkit}

The previous sections outlined the coalgebraic foundation for the finite state verification of name passing process calculi. It remains to show that this elegant theory can be used as a basis for the design and development of effective and usable verification toolkits. This section and the one following explore this issue by describing our experience in designing, implementing and experimenting a minimisation toolkit, called Mihda, for verifying finite state mobile systems represented in the $\pi$-calculus.

The Mihda toolkit cleanly separates facilities that are language-specific (parsing, transition system calculation) from those that are independent from the calculus notation (bisimulation) in order to facilitate modifications. The toolkit has been implemented in ocaml. Indeed, the partition refinement algorithm has been specified in a "type-theoretic" style and the underlying type system makes use of parametric polymorphism. The type system of ocaml offers all the necessary features for handling these kind of type. Figure 4 illustrates the modules of Mihda and their dependencies.

For instance, State is the module which provides all the structures for handling states and its main type defines the type of the states of the automata. Domination is the module containing the structures underlying bundle normalisation. The connections express typing relationships among the modules. For instance, since states in bundles and transitions must have the same type, then a connection exist between modules Bundle and Transitions.

Notice that the iterative construction of the minimal automaton is parameterised with respect to the modules of Figure 4. Indeed, the same algorithm can be applied to different kind of automata and bisimulation, provided that these automata match the constraints on types imposed by the software architecture. For instance, the architecture of Mihda has been exploited to provide minimisation of both HD-automata and ordinary automata (up to strong bisimilarity). 


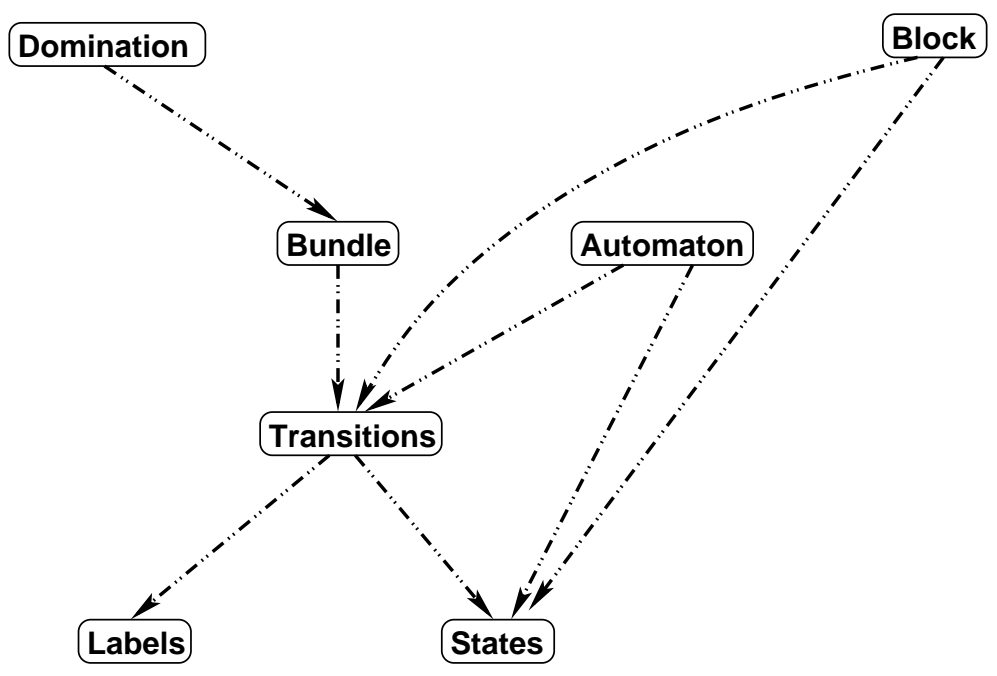

Fig. 3. Mihda Software Architecture

\subsection{The main cycle}

We have already pointed out that the iterative step of the minimisation algorithm can be represented in a functional style form as follows:

$$
h_{H_{i+1}}(q)=\operatorname{norm}\left\langle D_{H_{i}},\left\{\left\langle\ell, \pi, \sigma^{\prime} ; \sigma, h_{H_{i}}\left(q^{\prime}\right)\right\rangle \mid q \stackrel{\ell, \pi, \sigma}{\longrightarrow} q^{\prime}, \sigma^{\prime}: \Sigma_{H_{i}}\left(q^{\prime}\right)\right\}\right\rangle .
$$

Following equation (1), we can compute $h_{H_{i+1}}(q)$ through the following steps:

(a) determine the bundle of $q$ in the automaton;

(b) for each quadruple $\left\langle\ell, \pi, \sigma, q^{\prime}\right\rangle$ in this bundle, apply $h_{H_{i}}$ to $q^{\prime}$, the target state of the quadruple (yielding the bundle of $q^{\prime}$ in the previous iteration of the algorithm);

(c) left-compose symmetry $\sigma^{\prime} \in \Sigma\left(q^{\prime}\right)$ with $\sigma$;

(d) normalise the resulting bundle.

In the Mihda implementation the value of the i-th iteration (i.e. $h_{H_{i}}$ ) is stored in a list of blocks which are the most important data structures of Minda. They represent action of the functor on states of the automata and contain all those information for computing the iteration steps of the algorithm expressed in a set theoretic framework. Blocks represent both (finite) named functions and partitions of an automaton (at each iteration of the algorithm). Hence, at the last iteration a block corresponds to a state of the minimal automaton. A block has the following structure: 


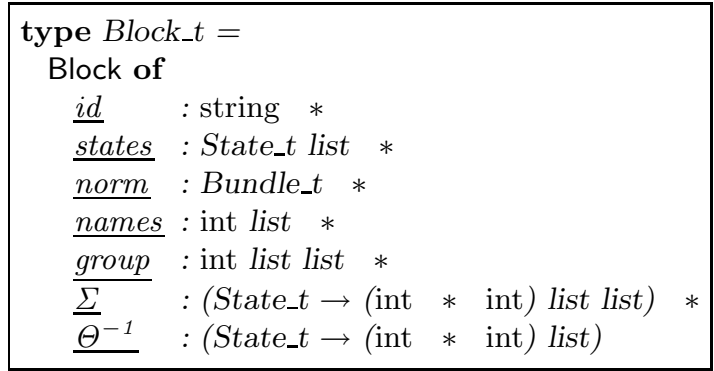

Field $\underline{i d}$ is the name of the block and is used to identify the block in order to construct the minimal automaton at the end of the algorithm. Field states contains the states which are considered equivalent with respect the equivalence relation used in the algorithm ${ }^{4}$ : In this case the early bisimulation relation. Remaining fields respectively represent

- the normalised bundle with respect to the block considered as state ( $\underline{\text { norm }})$,

- $\underline{\text { names }}$ is the list of names of the bundle in $\underline{\text { norm }}$,

- group is its group,

- the functions relative to the bundle $(\underline{\Sigma})$, last field, $\underline{\Theta^{-1}}$, is the function that, given a state $q$, maps the names appearing in $\underline{\text { norm }}$ into the name of $q$. Basically, $\Theta^{-1}(q)$ is the function which establishes a correspondence between the bundle of $q$ and the bundle of the corresponding representative element in the equivalence class of the minimal automaton.

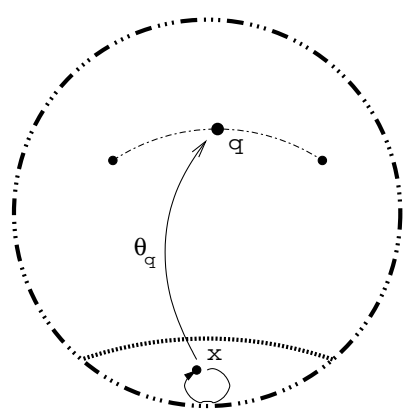

Fig. 4. Graphical representation of a block

We draw (some components of) a block as in Figure 4: The upper elements are the states in the block, while the element $\mathrm{x}$ is the "representative state", namely it is a graphical representation of the block as a state. For each state $q$ a function $\theta_{\mathrm{q}}$ maps names of $\mathrm{x}$ into the names of $\mathrm{q}$. Function $\theta_{\mathrm{q}}$ describes "how" the block approximates the state $\mathrm{q}$ at a given iteration. The circled arrow on $\mathrm{x}$ aims at

\footnotetext{
${ }^{4}$ We recall that Mihda is parametrised with respect to the equivalence relation.
} 
recording that a block also has symmetries on its names. Bundle $\underline{\text { norm }}$ of block $\mathrm{x}$ is computed by exploiting the ordering relations over names, labels and states.

A graphical representation of steps (a)-(d) above in terms of blocks is illustrated in Figure 5.

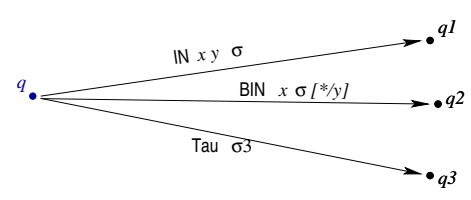

(a) Step 1

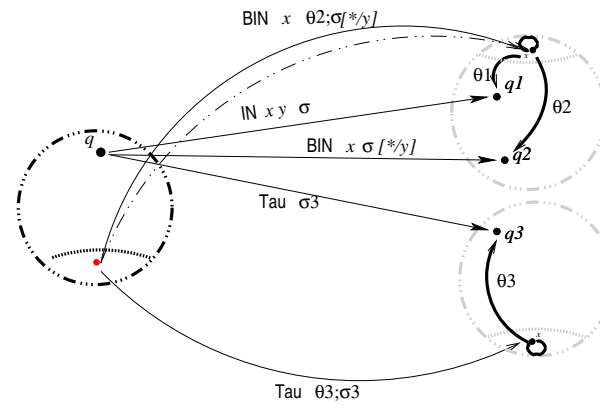

(c) Step 3

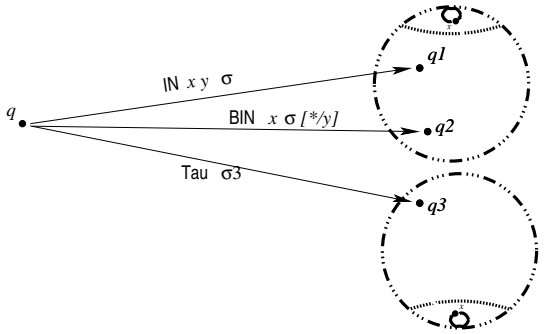

(b) Step 2

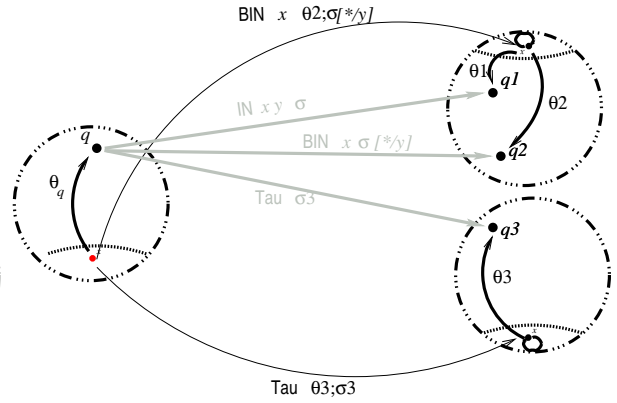

(d) Step 4

Fig. 5. Computing $h_{H_{i+1}}$

Step (a) is computed by the facility Automaton.bundle that filters all transitions of the automaton whose source corresponds to $q$. Figure 5 (a) shows that a state $\mathrm{q}$ is taken from a block and its bundle is computed.

Step (b) is obtained by applying facility Block.next to the bundle of q. The operation Block.next substitutes all target states of the quadruples with the corresponding current block and computes the new mappings (see Figure 5(b)). Step (c) does not seem to correctly adhere to the corresponding step of equation 1 . However, if we consider that $\theta$ functions are computed at each step by composing symmetries $\sigma$ 's we can easily see that $\theta$ functions exactly play the rôle of $\sigma$ 's. 
Finally, step (d) is represented in Figure 5(d) and is obtained via the function Bundle.normalise.

The main step of the minimisation algorithm is the function split that computes, at each iteration, the current partition (the list of blocks).

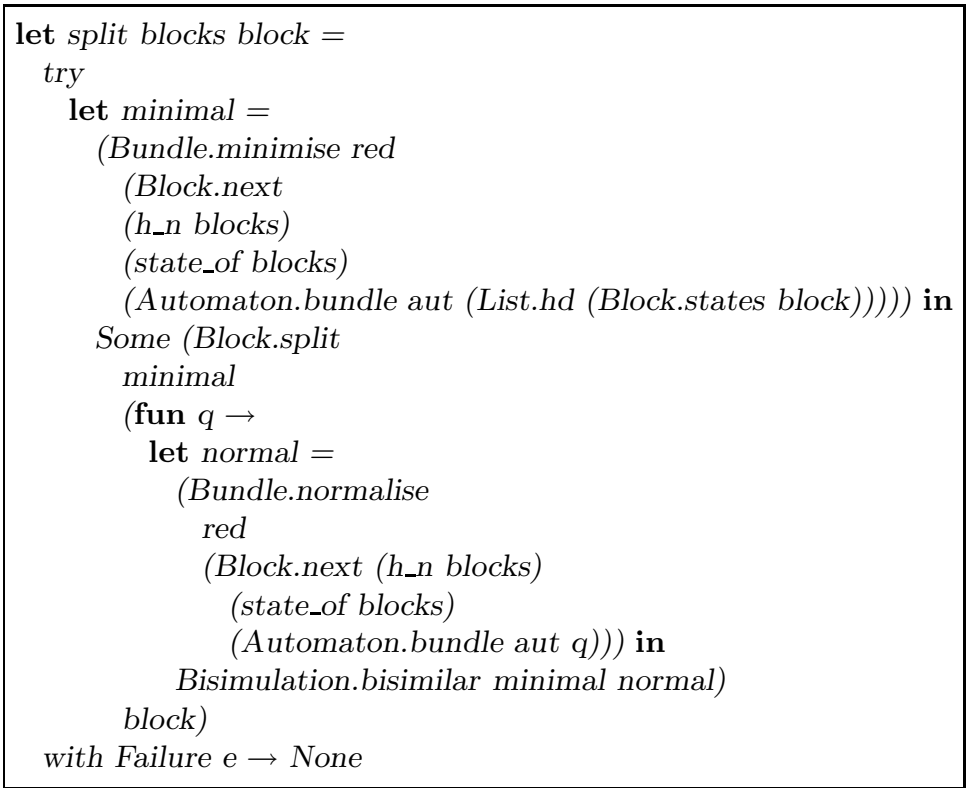

Let block be a block in the list blocks, function split computes minimal by minimising the reduced bundle of the first state of block. The choice of the state for computing minimal is not important: Without loss of generality, given two equivalent states q and q', it is possible to map names of q into names of q' preserving their associated normalised bundle if, and only if, a similar map from names of q' into names of q exists.

Once minimal has been computed, split invokes Block.split with parameters minimal and block, while the second argument of Block.split is a function that computes the current normalised bundle of each state in block and checks whether or not it is bisimilar to minimal. This computation is performed by function Bisimulation.bisimilar. If bisimilarity holds through $\theta_{\mathrm{q}}$ then Some $\theta_{\mathrm{q}}$ is returned, otherwise None is returned.

We are now ready to comment on the main cycle of Mihda reported in Figure 6 . Let $\mathrm{k}=$ (start, states, arrows) be a HD-automaton. When the algorithm starts, blocks is the list that contains a single block collecting all the states of the automata $\mathrm{k}$.

At each iteration, the list of blocks is splitted, as much as, possible by split_iter that returns a list of buckets which have the same fields of a block apart from the name, symmetries and the functions mapping names of destination states into names of source states. Essentially, the split operation checks if two states in a block are equivalent or not. States which are no longer equivalent to the representative element of the block are removed and inserted into 


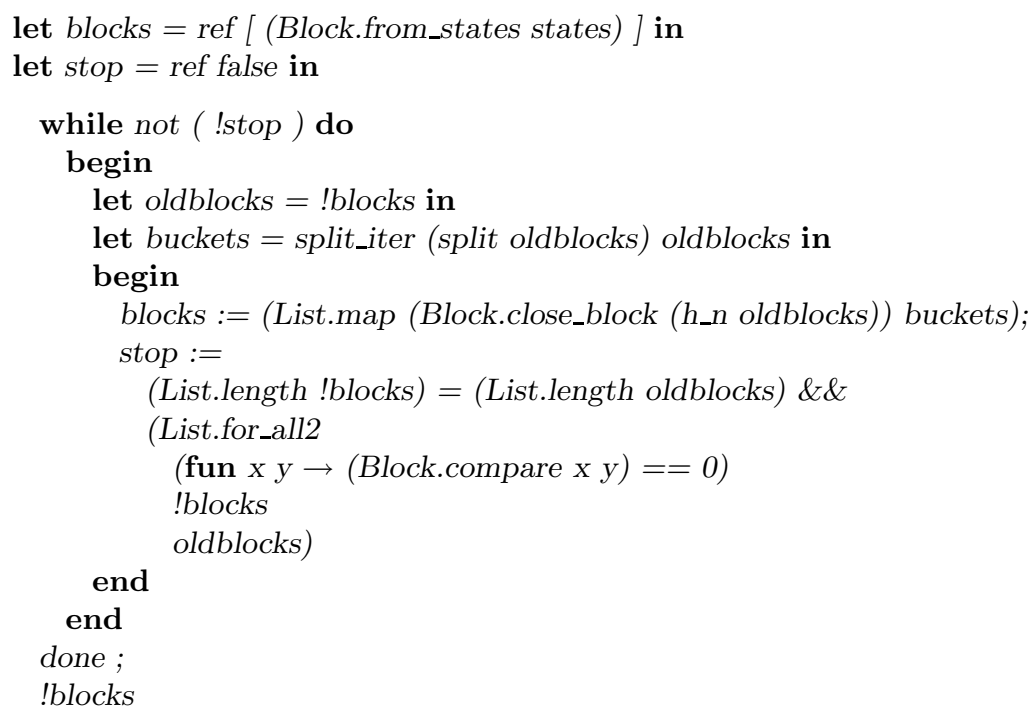

Fig. 6. The main cycle of Mihda

a bucket. Then, by means of Block.close_block, all buckets are turned into blocks which are assigned to blocks. Finally, the termination condition stop is evaluated. This condition is equivalent to say that a bijection can be established between oldblocks (that corresponds to $D_{i}$ ) and blocks (corresponding to $\left.D_{i+1}\right)$. Moreover, since order of states, names and bundles is always maintained along iterations, both lists of blocks are ordered. Hence, the condition reduces to test whether blocks and oldblocks have the same length and that blocks at corresponding positions are equal.

\section{$5 \quad$ Verifying Mobile Systems with Mihda}

In this section we discuss some experimental results of Mihda in the analysis of mobile systems. In particular, we consider the $\pi$-calculus specification of the Handover Protocol for Mobile Telephones borrowed from that given in [17] (which has been in turn derived from that in [13]).

The $\pi$-calculus specification of the GSM is

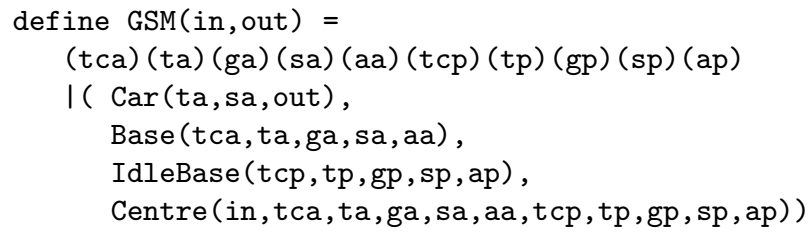

Centre receives messages from the environment on channel in; these input actions are the only observable actions performed by Centre. Module Car sends 


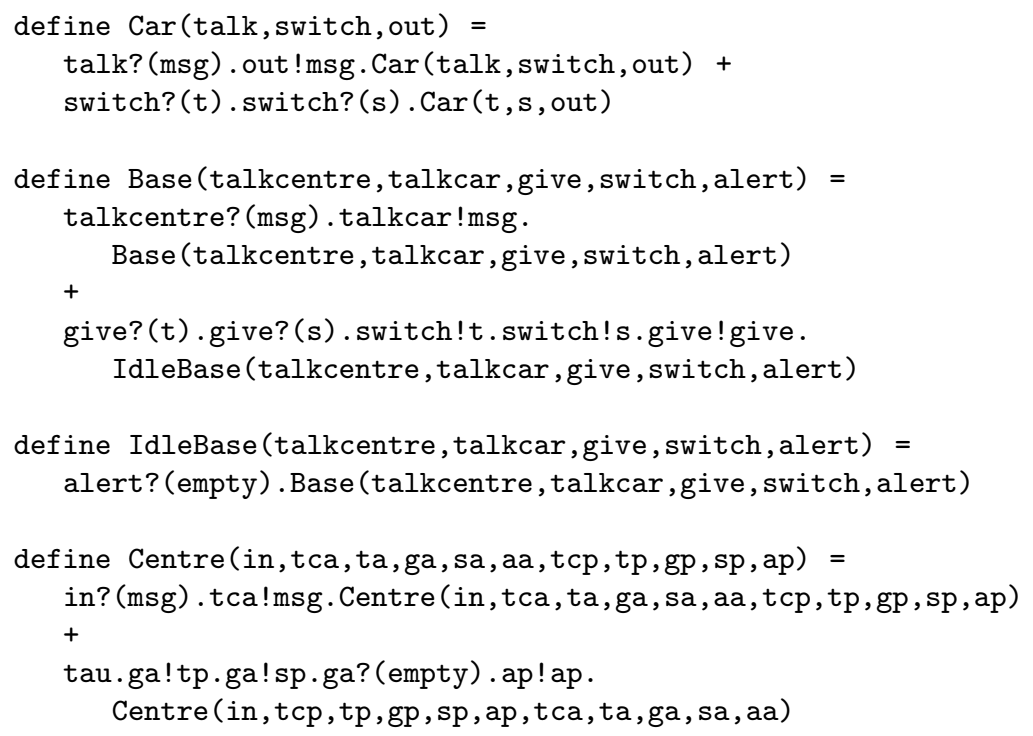

Table 3. $\pi$-calculus specification of GSM modules

the messages to the end user along the channel out; these outputs are the only visible actions performed by the Car. Modules Centre and Car interact via the base corresponding to the cell in which the car is located. The specification of modules Car, Base, IdleBase and Centre is reported in Table 3. The behaviour of the four modules is briefly summarised below:

- Car brings a MobileStation and travels across two different geographical areas that provide services to end users;

- Base and IdleBase are Base Station modules; they interconnect the MobileStation and the MobileSwitching Centre.

- Centre is a MobileSwitching centre which controls radio communications within the whole area composed by the two cells;

The protocol starts when Car moves from one cell to the other. Indeed, Centre communicates to the MobileStation the name of the base corresponding to the new cell. The communication of the new channel name to the MobileStation is performed via the current base. All the communications of messages between the MobileSwitching centre and the MobileStation are suspended until the MobileStation receives the names of the new transmission channels. Then the base corresponding to the new cell is activated, and the communications between the MobileSwitching centre and the MobileStation continue through the new base.

In Table 4 we report the results of Mihda on two different versions of the protocols. The first row of the table corresponds to the version discussed above. 


\begin{tabular}{|c||c|c|c|c|c|c|}
\hline Protocol & Time to compile & States & Transitions & Time to minimise & States & Transitions \\
\hline GSM small & $0 \mathrm{~m} 0.931 \mathrm{~s}$ & 211 & 398 & $0 \mathrm{~m} \mathrm{4.193 \textrm {s }}$ & 105 & 197 \\
\hline GSM full & $0 \mathrm{~m} \mathrm{8.186 \textrm {s }}$ & 964 & 1778 & $0 \mathrm{~m} 54.690 \mathrm{~s}$ & 137 & 253 \\
\hline
\end{tabular}

Table 4. Mihda at work

The second line gives the figures on a version of the GSM protocol that models the MobileSwitching and MobileStation modules in a more realistic way. Indeed, the 'full' version exploits a protocol for establishing whether or not the car is crossing the boundary of a cell and entering the other cell.

The results are obtained by running Mihda on a machine equipped with an AMD Athlon ${ }^{\mathrm{TM}} \mathrm{XP} 1800+$ dual processor with 1Giga RAM. The time for minimising the automata is very contained. The results on the GSM seem very promising. Indeed, the size of the minimal automata in terms of states and transitions is sensibly smaller than their non-minimised version. In the case of GSM small the size of the minimal automaton is the half or the automaton obtained by compiling the original specification; while, in version GSM full, states and transitions are reduced of a factor 8 .

\section{Conclusions}

This paper has provided an overview of a foundational model for the finite state verification of global computing systems and has showed how efficient tool supports can be derived from it.

In the future we plan to extend the Mihda toolkit with facilities to handle other notions of equivalences (e.g. open bisimilarity) and other foundational calculi for global computing (e.g. the asynchronous $\pi$-calculus, the fusion calculus). To improve efficiency, it could also be fruitful to incorporate supports for symbolic approaches based on Binary Decision Diagrams.

\section{References}

1. Peter Aczel. Algebras and coalgebras. In Roy Backhouse, Roland Crole and Jeremy Gibbons, editors, Algebraic and Coalgebraic Methods in the Mathematics of Program Construction, volume 2297 of LNCS, chapter 3, pages 79-88. Springer Verlag, April 2002. Revised Lectures of the Int. Summer School and Workshop.

2. Edmund M. Clarke and Jeanette M. Wing. Formal methods: state of the art and future directions. ACM Computing Surveys, 28(4):626-643, December 1996.

3. Jean Claude Fernandez. An implementation of an efficient algorithm for bisimulation equivalence. Science of Computer Programming, 13:219-236, 1990.

4. GianLuigi Ferrari, Ugo Montanari, and Marco Pistore. Minimizing transition systems for name passing calculi: A co-algebraic formulation. In Mogens Nielsen and Uffe Engberg, editors, FOSSACS 2002, volume LNCS 2303, pages 129-143. Springer Verlag, 2002. 
5. Marcelo Fiore, Gordon G. Plotkin, and Daniele Turi. Abstract syntax and variable binding. In 14th Annual Symposium on Logic in Computer Science. IEEE Computer Society Press, 1999.

6. Murdoch J. Gabbay and Andrew M. Pitts. A new approach to abstract syntax involving binders. In 14th Annual Symposium on Logic in Computer Science. IEEE Computer Society Press, 1999.

7. Bart Jacobs and Jan Rutten. A tutorial on (co)algebras and (co)induction. Bulletin of the EATCS, 62:222-259, 1996.

8. Paris C. Kanellakis and Scott A. Smolka. Ccs expressions, finite state processes and three problem of equivalence. Information and Computation, 86(1):272-302, 1990.

9. Robin Milner. Commuticating and Mobile Systems: the $\pi$-calculus. Cambridge University Press, 1999.

10. Robin Milner, Joachim Parrow, and David Walker. A calculus of mobile processes, I and II. Information and Computation, 100(1):1-40,41-77, September 1992.

11. Ugo Montanari and Marco Pistore. History dependent automata. Technical report, Computer Science Department, Università di Pisa, 1998. TR-11-98.

12. Ugo Montanari and Marco Pistore. $\pi$-calculus, structured coalgebras and minimal hd-automata. In Mathematical Foundations of Computer Science 2000, volume 1893. Springer, 2000.

13. Fredrik Orava and Joachim Parrow. An algebraic verification of a mobile network. Formal Aspects of Computing, 4(5):497-543, 1992.

14. Marco Pistore. History dependent automata. PhD thesis, Computer Science Department, Università di Pisa, 1999.

15. Andrew M. Pitts and Murdoch J. Gabbay. A metalanguage for programming with bound names modulo renaming. In Mathematics of Program Construction, 5th International Conference, MPC2000, volume 1837. Springer, 2000.

16. Davide Sangiorgi and David Walker. The $\pi$-calculus: a Theory of Mobile Processes. Cambridge University Press, 2002.

17. Björn Victor and Faron Moller. The Mobility Workbench - a tool for the $\pi$ calculus. In David Dill, editor, Proceedings of CAV'94, volume 818 of Lecture Notes in Computer Science, pages 428-440. Springer-Verlag, 1994. 